\title{
Erratum to: Formal Modelling and Verification of IEC61499 Function Blocks with Abstract State Machines and SMV - Execution Semantics
}

\author{
Sandeep Patil ${ }^{1(\otimes)}$, Victor Dubinin ${ }^{2}$, and Victor Vyatkin ${ }^{1,3}$ \\ ${ }^{1}$ Luleå University of Technology, Luleå, Sweden \\ sandeep.patil@ltu.se, vyatkin@ieee.org \\ ${ }^{2}$ Penza State University, Penza, Russia \\ victor_n_dubinin@yahoo.com \\ ${ }^{3}$ Aalto University, Espoo, Finland
}

\section{Erratum to: \\ Chapter 20 in: $X$. Li et al. (Eds.) \\ Dependable Software Engineering \\ DOI: 10.1007/978-3-319-25942-0_20}

In the original version, the name of the last author is incorrect. Instead of "Victor Vyatkin" it should be read as "Valeriy Vyatkin". 